\title{
Desensitization protocols for prospective pediatric renal transplant recipients
}

\author{
Nizam Mamode ${ }^{1,2} \cdot$ Stephen D. Marks $^{2}$ \\ Received: 18 April 2016 / Accepted: 13 May 2016 / Published online: 6 June 2016 \\ (C) IPNA 2016
}

\begin{abstract}
Desensitization protocols should be considered for children with positive crossmatches awaiting renal transplantation. Children are sensitized usually due to previous renal (and/or other solid-organ) transplants but can be from administration of blood and/or platelet transfusions, infections, and immunizations (as sensitization from pregnancy is a rare occurrence in pediatric patients). However, the definition of HLA-incompatible (HLAi) renal transplantation in the literature varies and is best considered only when there is a positive cross-match (positive baseline flow cytometric cross-match or positive complement-dependent cytotoxic cross-match). Renal transplantation where the recipient has donor-specific antibodies (DSA) but a negative cross-match should not fall into this category, although they are higher risk.
\end{abstract}

Keywords HLA incompatible · Renal transplantation · Children $\cdot$ Panel reactive antibody $\cdot$ Cumulative reaction frequency

\section{Introduction}

Around a third of adults presenting for transplantation have an antibody incompatibility [1] and the number of HLAi renal

Nizam Mamode

nizam.mamode@gstt.nhs.uk

1 Department of Transplantation, Guy's and St Thomas' NHS Foundation Trust, London SE1 9RT, UK

2 Department of Paediatric Nephrology, Great Ormond Street Hospital for Children NHS Foundation Trust, Great Ormond Street, London WC1N 3JH, UK transplants in adults has risen significantly in the last decade [2]. However, the number of children undergoing renal transplantation when the baseline cross-match is positive has remained largely unchanged; indeed, the United Network for Organ Sharing (UNOS) data shows no increase in deceased donor transplantation rates in children with panel reactive antibody (PRA) $>98 \%$ from 2004 to 2014, but a quadrupling in transplantation rates in those with little or no antibody [3]. Given the increase in transplantation of small children, it might be expected that the numbers presenting for a second transplant, who are likely to be sensitized, will increase further.

Despite this, reports of HLAi renal transplantation in children are essentially non-existent. Reasons for this are unclear, but likely to be multifactorial. There may be few HLAi renal transplants performed, possibly with poorer clinical outcomes (with respect to patient and renal allograft survival), and thus publication bias. Clinicians may perceive that algorithms which favor children in national allocation schemes mean that the chances of a deceased donor organ are high. However, in the UK, children with a cumulative reaction frequency $(\mathrm{CRF})>85 \%$ (which means antibodies against $85 \%$ of the last 10,000 deceased donors in the United Kingdom) account for $8 \%$ of the waiting list, but wait on average three and half years, which is four times longer than un-sensitized children (UK registry data). Centers may be concerned that children have both a higher risk of rejection, and a higher risk of infective complications (with the possibility of Epstein-Barr viral-induced post-transplant lymphoproliferative disorder). There are also relatively few large pediatric centers, so that cases may be seen infrequently and expertise may be narrow. Finally, antibody removal protocols may be considered practically difficult in small children by some centers. 


\section{Desensitization protocols}

The manuscript by Priojsakul et al. [4] is therefore a welcome addition to the literature. Unfortunately, there are a number of concerns with this paper. We feel that living and deceased donor HLAi renal transplantation should be considered separately. The former is defined by the presence of a positive baseline cross-match, whether complement dependent cytotoxic (CDC) or flow-cytometric, and the aim is to reduce antibody pre-transplant in order to achieve a negative crossmatch. The presence of donor-specific antibodies (DSA) in the context of a negative cross-match may confer a higher immunological risk, but would not require pre-transplant antibody removal and should have a high rate of success. In deceased donation, the issue is that the presence of high levels of HLA Ab either preclude an offer of a transplant, or result in a positive cross-match immediately prior to transplantation, when antibody removal is difficult. For these patients, the aim is to find therapies that decrease CRF or allow transplantation across a positive cross-match.

Priojsakul and colleagues [4] describe four cases, of which two were living donors: one had a positive cross-match with a low level of DSA and one a negative baseline cross-match and no significant DSA. It is unclear whether a subsequent positive B cell flow cytometric cross-match could have been due to autoreactivity or administration of intravenous immunoglobulin (IVIg). Both underwent an intensive regimen including 10 days of bortezomib, two doses of rituximab, IVIg and plasmapheresis. Although short-term outcomes were good, we would consider this degree of immunosuppression unjustified, particularly in a patient with little or no antibody. The effect on antibody levels pre-transplant is a little unclear, but intravenous rituximab would not be expected to lower these. The other two cases both concern deceased donation: one patient was in fact 18 years of age at the time with high PRA. Again both patients received bortezomib, rituximab, IVIg, with one patient also receiving induction with anti-thymocyte globulin therapy. In the first patient, the desensitization protocol made little difference to the HLA antibody profile, while in the second there was a reduction, which resulted in a later compatible deceased donor transplant. Again in both cases, outcomes were essentially good.

\section{Options for highly sensitized children}

There are little data in the literature about the management of highly sensitized children. What should we then make of this report? Firstly, the protocols used are unusual, and we would view them as excessive. In our institution, a living donor pair with DSA mean fluorescence intensity (MFI) of under 5,000 would undergo renal transplantation following a single session of plasmapheresis and administration of IVIg $(0.5 \mathrm{~g} / \mathrm{kg})$, with alemtuzumab induction [5]. There would be no justification for desensitization in the context of a negative cross-match without DSA. Secondly, in deceased donation, the issue with desensitization is one of timing - a reduction in antibody needs to be sustained until an organ offer occurs. In this report, in only one case did a reduction occur, and transplantation occurred 8 months later. It is therefore difficult to know whether such a sustained reduction was due to the therapy, since fluctuations in antibody levels may occur normally.

There is increasing evidence in the literature that kidney transplant offers significant survival and quality-of-life advantages compared to dialysis, but that waiting times are long with limited options for highly sensitized patients $[6,7]$. Adults who receive kidney transplants from HLAi live donors have a substantial survival benefit as compared to patients who do not undergo transplantation and those who wait for transplants from deceased donors $[8,9]$.

\section{Conclusions}

We would recommend that centers not performing pediatric HLAi renal transplantation should have a formal referral process to centers that do perform these higher-risk transplants. We would advocate that due to increased complications, entry to kidney paired schemes (such as National Living Donor Kidney Sharing Scheme in the United Kingdom by National Health Service Blood and Transplant) should be considered first, and we normally list patients on three or four occasions. However, those with lower immunological risk with clinical urgency (such as running out of dialysis access) could proceed to HLAi renal transplantation earlier.

In summary, it is important for the pediatric transplant community to consider HLAi renal transplantation, but this paper raises more questions than it answers.

Acknowledgments This project was supported by the National Institute for Health Research (NiHR) Biomedical Research Centres based at Guy's and St Thomas' National Health Service (NHS) Foundation Trust and King's College London as well as Great Ormond Street Hospital for Children NHS Foundation Trust and University College London. The views expressed are those of the authors and not necessarily those of the NHS, the NiHR of the Department of Health.

\section{Compliance with ethical standards}

Conflict of interest The author(s) declare that they have no competing interests.

\section{References}

1. Segev DL, Gentry SE, Warren DS, Reeb B, Montgomery RA (2005) Kidney paired donation and optimizing the use of live donor organs. JAMA 293(15):1883-1890 
2. http://www.odt.nhs.uk/uk-transplant-registry/annual-activity-report/

3. OPTN/SRTR Annual Data Report 2014. Am J Transpl 2016 S2;37

4. Pirojsakul K, Desai D, Lacelle C, Seikaly MD (2015) Management of sensitized patients prior to renal transplants: a single center experience. Pediatr Nephrol. doi:10.1007/s00467-015-3295-Z

5. Adamusiak A, Stojanovic J, Shaw O, Vaughan R, Sebire NJ, Drage M, Kessaris N, Marks SD, Mamode N (2016) Desensitisation strategy enabling HLA-antibody incompatible living donor renal transplantation of highly sensitised children. Am J Transplant 16(S1):50-51

6. Keith DS, Vranic GM (2016) Approach to the highly sensitized kidney transplant candidate. Clin J Am Soc Nephrol 11(4):684-693

7. Iyer HS, Jackson AM, Zachary AA, Montgomery RA (2013) Transplanting the highly sensitized patient: trials and tribulations. Curr Opin Nephrol Hypertens 22(6):681-688
8. Orandi BJ, Luo X, Massie AB, Garonzik-Wang JM, Lonze BE, Ahmed R, Van Arendonk KJ, Stegall MD, Jordan SC, Oberholzer J, Dunn TB, Ratner LE, Kapur S, Pelletier RP, Roberts JP, Melcher ML, Singh P, Sudan DL, Posner MP, El-Amm JM, Shapiro R, Cooper M, Lipkowitz GS, Rees MA, Marsh CL, Sankari BR, Gerber DA, Nelson PW, Wellen J, Bozorgzadeh A, Gaber AO, Montgomery RA, Segev DL (2016) Survival benefit with kidney transplants from HLA-incompatible live donors. N Engl J Med 374(10):940-950

9. Rostaing LP, Malvezzi P (2016) HLA-incompatible kidney transplantation - worth the risk? N Engl J Med 374(10):982-984 